\title{
Chédiak-Higashi syndrome
}

INSERM

\section{Source}

INSERM. (1999). Orphanet: an online rare disease and orphan drug data base. ChédiakHigashisyndrome. ORPHA:167

Chédiak-Higashi syndrome (CHS) is a rare severe genetic disorder generally characterized by partial oculocutaneous albinism (OCA, see this term), severe immunodeficiency, mild bleeding, neurological dysfunction and lymphoproliferative disorder. A classic, early-onset form and an attenuated, later-onset form (Atypical CHS; see this term) have been described. 